
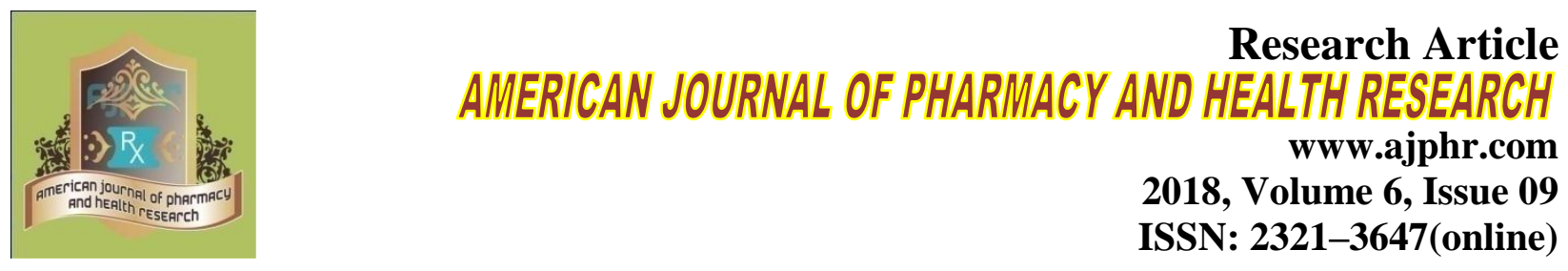

\title{
Case Report on Wellens Syndrome
}

\author{
Assem Babbar ${ }^{* 1}$, Rahul Rawat ${ }^{1}$, Ankit Sharma1, Prashant Mathur ${ }^{1}$ \\ 1. SGRRITS, Patel Nagar, Dehradun-248001, Uttarakhand
}

\begin{abstract}
Wellens syndrome is a pattern of electrocardiographic T-wave changes associated with critical, proximal left anterior descending (LAD) artery stenosis. We are presenting a case of wellens syndrome where 58 years old male patient admitted in the hospital with intermittent chest pain and dryness of mouth from last one month. He was diagnosed with Wellens Syndrome by ECG findings and treatment started accordingly. This syndrome may lead to major infarction if left unrecognized or untreated.
\end{abstract}

Key word: Wellens syndrome, LAD, ECG, Myocardial Infarction. 


\section{INTRODUCTION}

Wellens syndrome is a pattern of electrocardiographic T-wave changes associated with critical, proximal left anterior descending (LAD) artery stenosis. The syndrome is also referred to as LAD coronary T-wave syndrome. Syndrome criteria include T wave changes plus a history of anginal chest pain without serum marker abnormalities, patients lack $Q$ waves and significant ST-segment elevation, such patients show normal precordial R-wave progression. The natural history of Wellens syndrome is anterior wall acute myocardial infarction. The T-wave abnormalities are persistent and may remain in place for hours to weeks, the clinician likely will encounter these changes in the sensation-free patient. With definitive management of the stenosis, the changes resolve with normalization of the electrocardiogram ${ }^{1}$. If left untreated, most of the patients will develop into extensive anterior infarction, even death. Spasm-related angina sometimes shows similar ECG changes, and calcium channel blockers and long-acting nitrates, not the b-blockers and coronary interventions, are the mainstream of the therapy ${ }^{2}$.

\section{Case Report}

A 58year old male presented with intermittent chest pain and dryness of mouth from last one month. His pain has occurred mostly in the morning and at night, associated with excessive sweating, radiating to scapulas and relieving spontaneously. He had history of CAD and 40years of tobacco or smoking (50 biddies per day) but physical examination was normal. Laboratory examinations like complete blood count (CBC), electrolytes and renal function was normal. Cardiac enzymes were also normal. In ECG there were findings of Sinus Bradycardia, Abnormal Q wave, Anteroseptal Myocardial Infarction, ST segment deviation, T Wave abnormality (I, aVL, V2, V3, V4, V5, V6) Troponin I was found as 0.09ng/ml. He was diagnosed with Wellens Syndrome and given dual anti-platelet (oral aspirin and clopidogrel) and oral statin (atorvastatin) stat. The patient was brought to angiography laboratory immediately and 60 - 65\% LAD occlusion was observed. The patient was discharged from the hospital without any complication after angiographic intervention.

\section{RESULTS AND DISCUSSION}

T wave inversion is the most common ECG finding on the patients who are evaluated for the possible acute coronary syndrome in the emergency department (30\%) and generally interpreted as nonspecific ST-T deviations. Wellens Syndrome refers to the two different types of T wave pattern that are seen at the painless period. In Type A, which is the commonest form (75\%), there are deep negative T waves in leads V2 and V3 as in our first case. In type B (25\%), there are 
biphasic T waves typically observed in V2 and V3 as we have seen in our second case. This type of $\mathrm{T}$ wave deviations can also be observed in the other precordial leads depending on the proximity of the lesion in the associated artery. In addition to the $\mathrm{T}$ wave deviations in V2\& V3, there might be some extra findings in V4 (three out of four cases) and in V1 (two out of three cases). Studies also showed that these variances also might be seen in leads V5, V6 even the possibility is rare rat $^{3,4}$.

Clinical and electrocardiographic diagnostic criteria of Wellens' Syndrome are as follows ${ }^{3,4}$ -

- Negative and symmetrical deep T waves in V2 and V3 (rarely in V1,V4,V5 and V6) or Biphasic T waves in V2 and V3

- Isoelectric ST segment or mild elevation (1 mm)

- Absence of precordial Q waves

- History of angina

- Observation of findings in the painless period

- Normal or mildly elevated cardiac enzymes

Wellens patients are at high risk of myocardial infarction and sudden death, early detection of the distinct wellens ECG patterns will enable physicians to intervene and help prevent extensive anterior wall infarctions from occurring 5 .

\section{CONCLUSION}

We conclude that ECG is a cost - effective and inevitable biomarker for diagnosis of wellens syndrome. Patient with the history of MI or angina shows T wave abnormality in the ECG of wellens syndrome, may lead to sudden death if the patient passes any cardiac stress test. The syndrome may lead to major infarction if left unrecognized or untreated.

\section{REFERENCES}

1. Rhinehardt Joseph, D Brady J. William et al "Electrocardiographic manifestations of wellens syndrome”, American Journal of Emergency Medicine”, 2002 November, volume 20, issue 7 .

2. Abulaiti Alimeyiang, Aini Renaguli et al "Clinical Case Report Based Study - A special case of wellens syndrome", Journal of Cardiovascular Disease Research, 2013 February $27,51-54$.

3. Ozdemir Serdar, Ozturk Cimilli Tuba et al "Case report wellens syndrome - Report of two cases", Turkish Journal of Emergency Medicine, 2014 July 31, 179 - 181. 
4. Raheja Prafull, Sekhar Aravind et al "Wellens syndrome over the past three decades", Journal of Crdiovascular Medicine, 2013 February 5, 1-2.

5. MD Parikh S. Kishan, Agarwal Rajiv, MD Mehrotra K. Amit "Case report - wellens syndrome a life saving diagnosis”, American Journal of Emergency Medicine, 2012, 30, 255.e3 - 255.e5.

AJPHR is

Peer-reviewed

monthly

Rapid publication

Submit your next manuscript at editor@ajphr.com / editor.ajphr@gmail.com 